\title{
Analysis of Combining Ability for Early Maturity and Yield in Rice (Genus: Oryza) at the Kenyan Coast
}

\author{
Al-Imran Dianga $\mathbb{D}^{2},{ }^{2}$ Kamau W. Joseph $\mathbb{D}^{1},{ }^{1}$ and Ruth N. Musila $\mathbb{D}^{3}$ \\ ${ }^{1}$ Department of Plant Sciences, Kenyatta University, P. O. Box 43844-00100, Nairobi, Kenya \\ ${ }^{2}$ Department of Plant Sciences, Kenyatta University, P. O. Box 43844-00100, Nairobi, Kenya \\ ${ }^{3}$ Kenya Agricultural and Livestock Research Organization, P. O. Box 2981-0300, Kerugoya, Kenya \\ Correspondence should be addressed to Al-Imran Dianga; alimrandianga@gmail.com
}

Received 18 February 2020; Revised 23 April 2020; Accepted 18 September 2020; Published 22 October 2020

Academic Editor: Mehdi Rahimi

Copyright (c) 2020 Al-Imran Dianga et al. This is an open access article distributed under the Creative Commons Attribution License, which permits unrestricted use, distribution, and reproduction in any medium, provided the original work is properly cited.

\begin{abstract}
The main challenges facing rain-fed rice farming in Kilifi County at the Kenyan coast are inadequate and erratic rainfall, inadequate skills, and poorly developed infrastructure. Of great importance is erratic rainfall that tends to depress towards the end of long rain season, leading to crop failure. Combining ability analysis is one of the most valuable tools used to ascertain gene action effects and help in selecting desirable parents for making crosses and coming up with high yielding and early maturing lines. Combining ability for early maturity and yield has not been studied at the Kenyan coast. This study aimed at determining and identifying good, general, and specific combiners for selecting better parents and better cross combinations in rice crops for developing high yield and short duration lines in rain-fed rice farming. Seven lines were subjected to half-diallel mating design at the Kenya Agricultural and Livestock Research Organization (KALRO), Mtwapa, and at Bahari in Kilifi town. Evaluation for general combining ability (GCA) and specific combining ability (SCA) analysis was done. Combining ability variance and GCA and SCA effects were determined. Based on GCA effects, best parent for early maturity was Dourado Precoce, while for yield, Supaa, Komboka, and NERICA 10. SCA estimates indicated that best crosses for yield were D/S, D/N1, and K/N10, while the best performing cross for early maturity was D/N1.
\end{abstract}

\section{Introduction}

In Kenya, cultivation of rice is done in two ecosystems that are categorized based on water regimes basis: irrigated and rain-fed. The irrigated ecosystem produces $80 \%$ of cultivated rice, and only $20 \%$ comes from rain-fed ecosystem. Irrigated rice is grown in Kirinyaga, Kwale, Kilifi, and Tana River counties, while rain-fed farming is mainly practised in Busia, Kakamega, and Kisumu Counties [1]. Rice consumption in Kenya has been increasing at an annual rate of $12 \%$ compared to $1 \%$ for maize and $4 \%$ for wheat. This has been attributed to changes in eating habits especially in urban populations. In 2017 , rice consumption in the country was approximated at 700,000 metric tons seven times more than annual production range of 100,000 metric tons. The deficit was met through imports of about 600,000 metric tonnes in 2017 [2].

There is potential to develop about 540,000 ha of irrigated land and 1.0 million ha in rain-fed ecology for rice production in Kenya [1]. Irrigated rice productivity in Kenya is estimated at about $4-6 \mathrm{tha}^{-1}$, and rain-fed productivity is about $1 \mathrm{t} \mathrm{ha} \mathrm{h}^{-1}$ which are below optimum production potential of about $10 \mathrm{tha}^{-1}$ and $7 \mathrm{tha}^{-1}$ for irrigated and rainfed rice, respectively [1].

Combining ability analysis using Griffing [3] is most widely used as a biometric tool for identifying parental lines in terms of their ability to combine in hybrid combination. The resulting total genetic variation is partitioned into the variance effects of general combining ability (GCA) as a measure of additive gene action and specific combining ability (SCA), as a measure of additive gene action. The aim 
of this study was to detect the magnitude of both general and specific combining abilities for grain yield, maturity, and some agronomic characters in seven rice crosses using halfdiallel crosses.

\section{Materials and Methods}

Seven rice lines (Supaa, Komboka, Dourado Precoce, NERICA 1, NERICA 2, NERICA 4, and NERICA 10) were planted following half-diallel mating design during the long rain season of 2017 at KALRO, Mtwapa. Crosses were done in all possible combinations between each two of the seven parents without reciprocals to produce $21 \mathrm{~F}_{1}$ hybrids. The general combining ability (GCA) and specific combining ability (SCA) for yield and maturity among the seven lines and crosses were estimated. The $\mathrm{F}_{1}$ crosses were advanced to $\mathrm{F}_{2}$. In 2018 long rain season, the $\mathrm{F}_{2}$ crosses together with the parent lines were evaluated at KALRO, Mtwapa, and Bahari in Kilifi. Seeds of the twenty-one $\mathrm{F}_{2}$ hybrids and the seven parents were sown in a randomized complete block design (RCBD) with three replications. Each genotype was planted in a plot of $1.4 \mathrm{~m}$ by $1 \mathrm{~m}$ replicate with $20 \mathrm{~cm} \times 20 \mathrm{~cm}$ spacing within the row and $20 \mathrm{~cm}$ between the rows. Two seedlings were sown per hill that was later thinned to one.

Data were recorded on 20 individual plants chosen at random from each plot excluding border rows, and the last row on the plot was considered the border row. The following characteristics: days to maturity (50\% flowering), plant height $(\mathrm{cm})$, panicle length $(\mathrm{cm}), 1000$ grain weight $(\mathrm{g})$, and biomass $(\mathrm{g})$ were measured. Other characteristics included grain yield $\left(\mathrm{t} \mathrm{ha}^{-1}\right)$, brown rice length $(\mathrm{mm})$, brown rice width $(\mathrm{mm})$, number of tillers, number of unproductive tillers, number of fertile spikelet's per panicle, and number of unfertile spikelets per panicle following SES [4] protocols were also measured.

Analysis of variance was done on the combined data from the two sites. General combining ability and specific combining ability estimates were obtained by employing Griffing's [3] model 1(fixed model) method 2, expressed as

$$
X i j=u+g i+g j+s i j,
$$

where, $u$ is the overall mean of all entries in the diallel design, $g i$ is the general combining ability of the $i$ th parent, $g j$ is the general combining ability of the $j$ th parent, and sij is the specific combining ability between the $i$ th and $j$ th parent.

\section{Results and Discussion}

3.1. Analysis of Variance. Analysis of variance (Table 1) showed that variation among genotypes for yield, days to $50 \%$ flowering, plant height, number of tillers, unproductive tillers, unfertile spikelets, grain length, grain width, 1000 grain weight, and biomass were highly significant at $P \leq 0.001$. Fertile spikelet's, panicle weight, and panicle length were slightly significant at $P \leq 0.05$.

Parent mean squares showed significant variation at $P \leq 0.05$ for 1000 grain weight, grain width, panicle weight, panicle length, biomass, and fertile spikelets. Days to $50 \%$ flowering and unfertile spikelet's had the highest significance at $P \leq 0.001$. Crosses mean squares showed significant difference at $P \leq 0.05$ for all traits except 1000 grain weight. This suggests that parents and crosses were significantly different in mean performance.

Analysis of GCA variance showed significance for days to $50 \%$ flowering, biomass, plant height, tillers, fertile spikelets, grain length, unfertile spikelets, and yield. Grain width, unproductive tillers, panicle weight, and panicle length were insignificant. SCA variation reached significance for days to $50 \%$ flowering, 1000 grain weight, biomass, and unproductive tillers. Panicle weight, panicle length, unfertile spikelets, yield, grain width, grain length, fertile spikelets, and total tillers were insignificant.

3.2. Mean Performance of Genotypes. Genotypic performance (Table 2) showed that, among parents, Supaa and Komboka flowered late and hence are late maturing. Komboka and Supaa were the highest yielding parents, while NERICA 4 was the least yielding parent. Performance based on other traits showed Komboka was the best performer in terms of total number of fertile tillers and the highest fertile spikelets. Moreover, the best parent in panicle weight, grain width, and 1000 grain weight was Dourado Precoce. Lastly, the biomass yield was highest in Komboka and Supaa.

Genotypic performance among crosses showed that N1/ $\mathrm{N} 2, \mathrm{~N} 1 / \mathrm{N} 4$, and N10/S crosses took fewer days to $50 \%$ flowering, while plants from $\mathrm{S} / \mathrm{K}$ cross took longer. The highest yielding plants were $\mathrm{S} / \mathrm{K}, \mathrm{N} 4 / \mathrm{D}$, and N2/N10 crosses, while plants from N10/K, N2/D, and N4/S crosses were the least yielding crosses. Performance for other traits under study showed that plants from N2/S and N10/D crosses had the lowest unproductive tillers, while plants from N4/D and N1/N2 crosses had the greatest panicle weight. Furthermore, N4/D and N1/N2 crosses had the most fertile spikelets and the least unfertile spikelets. Rice grains from N4/D and N1/S had the largest width, while those of N4/D were the longest. In terms of 1000 grain weight, N2/D, N10/D, and N1/D crosses were the best performers, while $\mathrm{N} 1 / \mathrm{K}$ and $\mathrm{N} 2 / \mathrm{K}$ crosses produced the highest biomass yield. This result revealed the positive contribution of the genes to days to $50 \%$ flowering and grain and biomass yield. In particular, N4/D and N2/N10 yielded all other crosses and their parents. Plants from N10/S, N2/K, and D/S crosses also outperformed one of their parents as they matured earlier than one of their parents.

3.3. General Combining Ability (GCA) Effects. High positive GCA effects values would be of great interest in all characters except plant height, days to $50 \%$ flowering, unfertile spikelets, and unproductive tillers for which negative values become more useful from the breeder's point of view. Most parents showed positive GCA effects for days to $50 \%$ flowering except Dourado Precoce that showed significant negative GCA effects emerging as the best combiner ( $\mathrm{Ta}$ ble 3). Supaa was the poorest combiner in terms of days to $50 \%$ flowering because of the positive significant GCA effects. Significant GCA effects for grain yield were recorded for Supaa, Komboka, and NERICA 1. Komboka and Supaa 


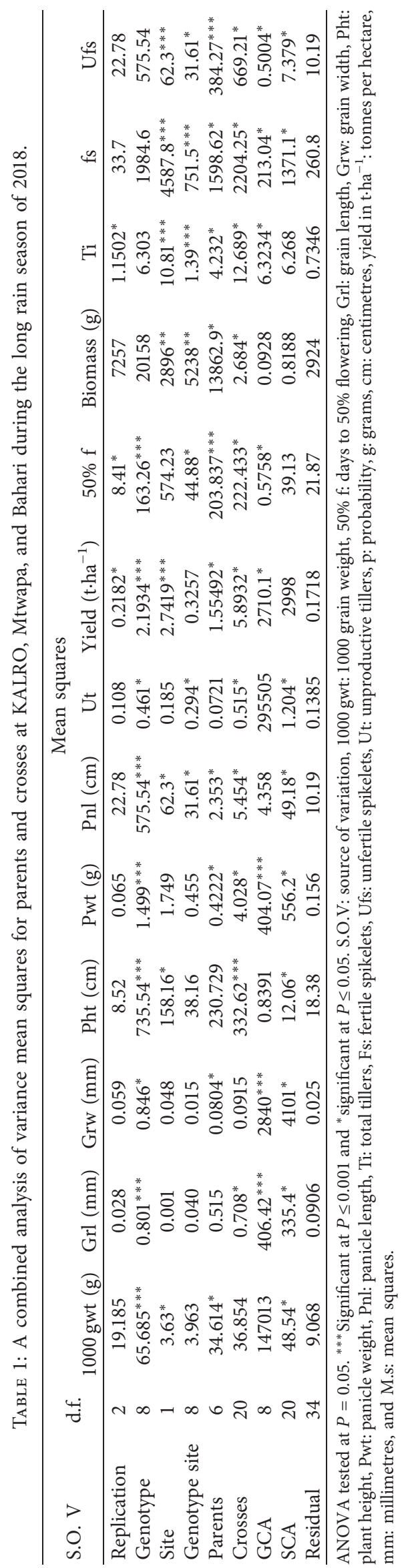


TABLe 2: Mean performance of genotypes for parents and crosses at KALRO, Mtwapa, and Bahari during the long rain season of 2018.

\begin{tabular}{|c|c|c|c|c|c|c|c|c|c|c|c|c|c|}
\hline Genotype & $\%$ f & Yield & $\begin{array}{l}\text { Pht } \\
(\mathrm{cm})\end{array}$ & $\mathrm{Ti}$ & Ut & $\begin{array}{l}\text { Pnl } \\
(\mathrm{cm})\end{array}$ & wt (g) & Fs & Ufs & $\begin{array}{c}\mathrm{Grl} \\
(\mathrm{mm})\end{array}$ & $\begin{array}{l}\text { Grw } \\
(\mathrm{mm})\end{array}$ & $\begin{array}{c}1000 \text { gwt } \\
(\mathrm{g})\end{array}$ & 10111 \\
\hline D. $p$ & $3 \mathrm{ab}$ & $5 b$ & $838 \mathrm{~b}$ & $5 b$ & $0.54 \mathrm{ab}$ & $23.43 \mathrm{bc}$ & $28 c$ & 127 & $17.3 \mathrm{ab}$ & $10.05 c$ & $3.005 c$ & $35.2 d$ & $56 \mathrm{~b}$ \\
\hline $\mathrm{D} / \mathrm{K}$ & $.7 \mathrm{ab}$ & $08 \mathrm{ab}$ & $2 \mathrm{ab}$ & $7.01 \mathrm{c}$ & $0.42 \mathrm{ab}$ & $4.01 \mathrm{bc}$ & $2.32 \mathrm{ab}$ & & & & & & $4 \mathrm{bc}$ \\
\hline $\mathrm{D} / \mathrm{S}$ & $8 \mathrm{ab}$ & $2.09 \mathrm{ab}$ & 76.4ab & $6.46 \mathrm{bc}$ & & $22.25 a$ & $2.47 \mathrm{ab}$ & $116.4 \mathrm{ab}$ & & & c & $32.7 c$ & \\
\hline Komboka & $85.8 \mathrm{c}$ & $4.0 \mathrm{c}$ & $75 \mathrm{ab}$ & $.28 \mathrm{~d}$ & $0.28 \mathrm{a}$ & $24.26 \mathrm{c}$ & $3.56 b c$ & $115.20 \mathrm{bc}$ & $30.9 b$ & $8.94 a$ & $.487 a$ & $24.2 \mathrm{a}$ & $19 b c$ \\
\hline NERICA 1 & $74.2 \mathrm{ab}$ & $2.61 \mathrm{ab}$ & $69 a$ & $5.29 \mathrm{~b}$ & $0.7 \mathrm{ab}$ & $23.53 \mathrm{bc}$ & $3.83 b c$ & & $12.5 \mathrm{a}$ & & & $31.2 b c$ & $51 \mathrm{ab}$ \\
\hline N1/D & $.2 \mathrm{ab}$ & $2.95 \mathrm{ab}$ & 73.3ab & $5.03 \mathrm{ab}$ & $0.78 \mathrm{ab}$ & $22.8 b c$ & $3.65 b c$ & $151.5 \mathrm{c}$ & $29 \mathrm{ab}$ & $9.81 b c$ & $3.319 \mathrm{~d}$ & & $320 a b$ \\
\hline $\mathrm{N} 1 / \mathrm{K}$ & $3 \mathrm{ab}$ & $2.21 \mathrm{ab}$ & $72.3 \mathrm{ab}$ & $6.53 b c$ & $1.07 \mathrm{~b}$ & $23.55 b c$ & $2.57 \mathrm{ab}$ & $\mathrm{bc}$ & $50.1 \mathrm{c}$ & & $2.716 b$ & $30.2 \mathrm{bc}$ & $453 c$ \\
\hline $\mathrm{N} 1 / \mathrm{N} 1$ & $8 \mathrm{ab}$ & $91 \mathrm{ab}$ & $71.4 \mathrm{ab}$ & $5.93 b c$ & $0.6 \mathrm{ab}$ & $3.76 b c$ & & & & & & & $3 \mathrm{ab}$ \\
\hline $\mathrm{N}$ & $3 a$ & $b$ & $73.8 \mathrm{ab}$ & & $0.53 \mathrm{ab}$ & c & & & & & & & \\
\hline (2) & & $2.69 \mathrm{ab}$ & $73 \mathrm{ab}$ & $5.95 b c$ & $0.83 \mathrm{ab}$ & & & & & & & $\mathrm{cd}$ & $330 \mathrm{ab}$ \\
\hline $\mathrm{N} 1 / \mathrm{S}$ & b & $50 \mathrm{Ob}$ & 750 & 501 & 0.46 & 2 & 24 & 135 & & & & & $362 b$ \\
\hline $\begin{array}{l}\text { NERICA } \\
10\end{array}$ & 7 & 3 & 74. & & 0 & & & 160 & & 8 & $2.681 b$ & 2 & $295 \mathrm{ab}$ \\
\hline $\mathrm{N} 10 / \mathrm{D}$ & $.5 \mathrm{ab}$ & $2.87 \mathrm{ab}$ & $.6 \mathrm{~b}$ & $.75 \mathrm{ab}$ & $0.68 \mathrm{ab}$ & $22.89 \mathrm{~b}$ & & & & & & & $88 \mathrm{ab}$ \\
\hline & & 72 & $72.1 \mathrm{ab}$ & $6.92 c$ & 11 & $\mathrm{bc}$ & & & & & & & bc \\
\hline & & & $73.2 \mathrm{ab}$ & $6.56 b c$ & & & & & & & & & \\
\hline NERICA 2 & $73.7 \mathrm{ab}$ & $3.13 b$ & $73 \mathrm{ab}$ & $5.66 \mathrm{~b}$ & $0.64 \mathrm{ab}$ & $21.66 \mathrm{ab}$ & & & 19 & & & $28.3 \mathrm{ab}$ & $1 \mathrm{ab}$ \\
\hline $\mathrm{N} 2 / \mathrm{D}$ & $71 \mathrm{ab}$ & $1.98 \mathrm{a}$ & 73.6ab & $5.88 \mathrm{bc}$ & $0.65 \mathrm{ab}$ & $24.32 \mathrm{bc}$ & $3.52 \mathrm{bc}$ & & & & & & $342 \mathrm{ab}$ \\
\hline $\mathrm{N} 2 / \mathrm{K}$ & 73ab & $2.04 \mathrm{ab}$ & 74.1ab & $8.07 \mathrm{~d}$ & $2.03 c$ & $23.98 b c$ & & $144.2 \mathrm{bc}$ & 81. & & & & $436 \mathrm{bc}$ \\
\hline & $72.7 \mathrm{ab}$ & & $66.5 \mathrm{a}$ & & $0.58 \mathrm{ab}$ & & & & & & & & $323 \mathrm{ab}$ \\
\hline $\mathrm{N} 2 / \mathrm{N} 4$ & $.5 \mathrm{ab}$ & $2.71 \mathrm{ab}$ & $72.4 \mathrm{ab}$ & $5.68 \mathrm{~b}$ & $0.74 \mathrm{ab}$ & $23.85 b c$ & $3.66 \mathrm{bc}$ & $\mathrm{bc}$ & 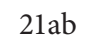 & $9.6 \mathrm{ab}$ & $\mathrm{bc}$ & $\mathrm{bcc}$ & $320 \mathrm{ab}$ \\
\hline $\mathrm{N} 2 / \mathrm{S}$ & $8 \mathrm{ab}$ & $2.52 \mathrm{ab}$ & $73.4 \mathrm{ab}$ & $4.27 \mathrm{a}$ & $0.49 \mathrm{ab}$ & $20.73 a$ & $2.52 \mathrm{ab}$ & $2 \mathrm{~b}$ & 40. & $\mathrm{ab}$ & & $3 a b$ & $381 b$ \\
\hline NERICA 4 & $.7 \mathrm{ab}$ & $2.82 \mathrm{ab}$ & 72ab & $4.92 \mathrm{ab}$ & $0.5 \mathrm{ab}$ & $23.71 b c$ & $3.78 b c$ & & $17.8 \mathrm{a}$ & & & $29.7 b$ & $327 \mathrm{ab}$ \\
\hline $\mathrm{N} 4 / \mathrm{D}$ & $71.3 \mathrm{ab}$ & $3.19 \mathrm{ab}$ & $78.4 \mathrm{~b}$ & $5.15 \mathrm{ab}$ & $0.47 \mathrm{ab}$ & & & & & & & & \\
\hline $\mathrm{N} 4 / \mathrm{K}$ & $79.3 \mathrm{bc}$ & $2.64 \mathrm{ab}$ & $84 \mathrm{~b}$ & $6.43 b c$ & $0.57 \mathrm{ab}$ & $23.65 b c$ & & & & & & & $435 b c$ \\
\hline N4/N10 & $69 a$ & $3.31 \mathrm{~b}$ & $72.5 \mathrm{ab}$ & $6.04 \mathrm{~b}$ & $0.65 \mathrm{ab}$ & $23.74 b c$ & $3.95 b c$ & $142.3 \mathrm{c}$ & $22.9 \mathrm{ab}$ & $9.6 \mathrm{bc}$ & $2.65 \mathrm{ab}$ & $33 \mathrm{bc}$ & $309 \mathrm{ab}$ \\
\hline $\mathrm{N} 4 / \mathrm{S}$ & & $1.87 \mathrm{ab}$ & $84.7 \mathrm{~b}$ & $6.81 b c$ & $0.87 \mathrm{ab}$ & $22.69 b c$ & $3.08 \mathrm{ab}$ & $136.2 \mathrm{~b}$ & $48.8 \mathrm{bc}$ & $9.48 \mathrm{~b}$ & $2.646 \mathrm{ab}$ & $27.8 \mathrm{ab}$ & $372 \mathrm{ab}$ \\
\hline S & 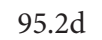 & & $93.2 \mathrm{c}$ & & & & & & & & & & $490 \mathrm{c}$ \\
\hline $\mathrm{S} / \mathrm{K}$ & $80.5 b c$ & $4.09 \mathrm{c}$ & $80.6 \mathrm{ab}$ & $6.68 \mathrm{bc}$ & $0.43 \mathrm{a}$ & $24.11 \mathrm{bc}$ & $2.64 \mathrm{ab}$ & $139.3 \mathrm{~b}$ & $37.7 \mathrm{~b}$ & $9.41 \mathrm{~b}$ & $2.597 \mathrm{ab}$ & $34 c$ & $329 a b$ \\
\hline
\end{tabular}

Mean values in the table denoted by different letters are significantly different at $P=0.05$. Two-way ANOVA, $50 \%$ f: days to $50 \%$ flowering, 100 gwt: 1000 grain weight, Grl: grain length, Grw: grain width, Pht: plant height, Pwt: panicle weight, Pnl: panicle length, Rt: reproductive tillers, Ti: total tillers, Fs: fertile spikelets, Ufs: unfertile spikelets, Ut: unproductive tillers, Mean separation Tukeys.

were found to be best combiner parents for yield, while NERICA 1 was the poorest parent combiner with significant negative GCA effects.

The best combiner for plant height was NERICA 1. For number of tillers, Supaa and Komboka showed significant positive GCA indicating they were the best combiners for a number of tillers. NERICA 2 was the poorest combiner with negative GCA effects. For unfertile spikelets, NERICA 10 had the most significant positive effects, thus emerging as the poorest combiner. Significant negative GCA was shown by NERICA 1 and Dourado Precoce which emerged as the best combiners. Dourado Precoce, Supaa, and NERICA 2 were the best combiners for grain length, while NERICA 10 was the poorest combiner. For biomass, Supaa and Komboka had the most significant positive GCA and were the best combiners, while NERICA 10 posed significant negative GCA effect and was the poorest combiner.

3.4. Specific Combining Ability (SCA) Effects. A significant deviation from zero in SCA of across would indicate high or low specific combining ability (SCA) depending on weather the sign is positive or negative. Significant negative SCA is desirable for all traits under study except plant height, days to $50 \%$ flowering, unfertile spikelets, and unproductive tillers. The positive SCA effects for days to $50 \%$ flowering were significant for $\mathrm{S} / \mathrm{K}, \mathrm{N} 1 / \mathrm{S}$, and $\mathrm{K} / \mathrm{N} 10$ crosses, thus emerging as the poorest performing crosses based on SCA effects (Table 4), while cross D/N1 with significant positive SCA effects was the best performer. The best yielding crosses were $\mathrm{D} / \mathrm{S}$ and N10/S crosses with high significant negative SCA effects, while N1/N2 was the poorest combiner with significant positive SCA effects.

Plants from K/N10 and D/S crosses were the best crosses in terms of plant height as they had positive significant SCA effects. N10/K and K/N2 crosses performed better in relation to unproductive tillers, while N1/N2 cross performed poorly as it had significant negative SCA effects. Performance based on panicle length indicated that $\mathrm{K} / \mathrm{N} 4$ and $\mathrm{D} / \mathrm{N} 10$ were the best crosses, while K/N2 was the poorest cross. Panicles from $\mathrm{K} / \mathrm{N} 2$ cross were the best in terms of SCA effects, as K/N2, N1/N2, and N10/S crosses were best for unfertile spikelets. For 1000 grain weight, N10/N2 cross was the best, as K/S, $\mathrm{N} 1 / \mathrm{S}$, and D/S crosses emerged as the best crosses in terms of biomass. Results from this study reveal that SCA effects and cross performance might closely relate. A cross with high SCA effects may not be the one with the highest genotypic performance and vice versa. The best way is to explore where 
TABLE 3: General combining ability effects for parents at KALRO, Mtwapa, and Bahari during the long rain season of 2018.

\begin{tabular}{lccccccccccccc}
\hline Parents & $\begin{array}{c}\text { Yield } \\
\left(\mathrm{t} \cdot \mathrm{ha}^{-1}\right)\end{array}$ & $50 \% \mathrm{f}$ & $\begin{array}{c}\text { Pht } \\
(\mathrm{cm})\end{array}$ & $\mathrm{Ut}$ & $\begin{array}{c}\text { Pnl } \\
(\mathrm{cm})\end{array}$ & $\begin{array}{c}\text { Pwt } \\
(\mathrm{g})\end{array}$ & Fs & Ufs & $\begin{array}{c}\text { Grl } \\
(\mathrm{mm})\end{array}$ & $\begin{array}{c}\text { Grw } \\
(\mathrm{mm})\end{array}$ & $\begin{array}{c}1000 \text { gwt } \\
(\mathrm{g})\end{array}$ & $\begin{array}{c}\text { Ti } \\
(\mathrm{g})\end{array}$ \\
\hline $\begin{array}{l}\text { Dourado } \\
\text { Precoce }\end{array}$ & 0.59 & $-0.67^{*}$ & 10.58 & -0.15 & 0.08 & 0.93 & -12.8 & $-16.65^{*}$ & $0.55^{*}$ & 0.234 & 3.97 & -0.86 & -2 \\
Komboka & $1.24^{*}$ & 11.83 & $-0.72^{*}$ & -0.41 & 0.91 & 0.21 & $41.4^{*}$ & -3.05 & -0.56 & -0.28 & -7.03 & $2.43^{*}$ & $46^{*}$ \\
Supaa & $1.79^{*}$ & $21.17^{*}$ & $17.48^{*}$ & -0.36 & -0.25 & -0.3 & -30.4 & 14.25 & $0.37^{*}$ & 0.161 & -0.53 & $0.83^{*}$ & $132^{*}$ \\
NERICA 1 & $-0.15^{*}$ & $0.17^{*}$ & $-6.62^{*}$ & 0.01 & 0.18 & 0.48 & -1.08 & $-21.45^{*}$ & -0.06 & 0.017 & -0.03 & -0.56 & -7 \\
NERICA 2 & -0.05 & 0.33 & -2.72 & -0.05 & -1.69 & 0.26 & 0.5 & -14.85 & $0.02^{*}$ & 0.043 & -2.93 & $-0.20^{*}$ & -57 \\
NERICA 4 & 0.052 & 0.33 & -3.72 & -0.19 & 0.36 & 0.43 & 11.1435 & -16.15 & -0.02 & 0.094 & -1.53 & -0.94 & -31 \\
NERICA 10 & 0.62 & 4 & -1.42 & -0.11 & 0.91 & 0.54 & 20.8 & $11.65^{*}$ & $-0.53^{*}$ & 0.103 & -3.43 & 0.025 & $-63^{*}$ \\
\hline
\end{tabular}

Significance tested at $P=0.05$, ${ }^{*}$ significant at $P \leq 0.05 .1000$ gwt: 1000 grain weight, $50 \%$ f: days to $50 \%$ flowering, Grl: grain length, Grw: grain width, Pht: plant height, Pwt: panicle weight, Pnl: panicle length, Ti: total tillers, Fs: fertile spikelets, Ufs: unfertile spikelets, Ut: unproductive tillers, g: grams, cm: centimetres, $\mathrm{t} \cdot \mathrm{ha}^{-1}$ : tonnes per hectare, and $\mathrm{mm}$ : millimetres.

Table 4: Specific combining ability effects for crosses at KALRO, Mtwapa, and Bahari during the long rain season of 2018.

\begin{tabular}{|c|c|c|c|c|c|c|c|c|c|c|c|c|c|}
\hline Cross & $\begin{array}{c}\text { Yield } \\
\left(\mathrm{t} \cdot \mathrm{ha}^{-1}\right)-\end{array}$ & $50 \% \mathrm{f}$ & $\begin{array}{l}\text { Pht } \\
(\mathrm{cm})\end{array}$ & $\mathrm{Ti}$ & Ut & $\begin{array}{l}\text { Pnl } \\
(\mathrm{cm})\end{array}$ & Pwt (g) & Fs & Ufs & $\begin{array}{c}\text { Grl } \\
(\mathrm{mm})\end{array}$ & $\begin{array}{l}\text { Grw } \\
(\mathrm{mm})\end{array}$ & $\begin{array}{l}1000 \mathrm{gwt} \\
\text { (g) }\end{array}$ & $\begin{array}{c}\text { Biomass } \\
(\mathrm{g})\end{array}$ \\
\hline $\mathrm{D} / \mathrm{N} 1$ & -0.37 & $3^{*}$ & -7.7 & 0.84 & 0.29 & -1.87 & -1.46 & -25.17 & 35.42 & -0.16 & 0.3 & -0.87 & -30.7 \\
\hline $\mathrm{K} / \mathrm{N} 1$ & -1.76 & -8.4 & 3.1 & 2.34 & 0.84 & -1.12 & -1.82 & -42.2 & 43.02 & -0.6 & 0.21 & 6.53 & 39.34 \\
\hline $\mathrm{D} / \mathrm{N} 10$ & -1.22 & -7.5 & -4.7 & -0.03 & 0.31 & $-2.51^{*}$ & 2.24 & -29.1 & 14.22 & 0.32 & 0.03 & 2.23 & 23.34 \\
\hline $\mathrm{K} / \mathrm{N} 10$ & -3.02 & $-17.3^{*}$ & $-5.8^{*}$ & -1.14 & $0.89^{*}$ & -2.35 & 1.78 & -54.8 & 11.9 & 1.067 & 0.37 & 8.53 & 29.34 \\
\hline $\begin{array}{l}\text { N1/ } \\
\text { N10 }\end{array}$ & -0.44 & -6.1 & 0 & 0.86 & 0.71 & -1.74 & -0.87 & -12.3 & 18.92 & 0.31 & 0.05 & 4.53 & 53.34 \\
\hline $\mathrm{D} / \mathrm{N} 2$ & -1.86 & -2.7 & -11.4 & 1.32 & 0.22 & 1.52 & -1.37 & 18.27 & 57.02 & -0.5 & -0.23 & 2.03 & 41.34 \\
\hline $\mathrm{K} / \mathrm{N} 2$ & -2.45 & -13.2 & 8.9 & -0.44 & $1.86^{*}$ & $0.35^{*}$ & $-1.99^{*}$ & -38.6 & $31.9^{*}$ & 0.53 & 0.14 & 8.73 & $72.34^{*}$ \\
\hline $\mathrm{N} 1 / \mathrm{N} 2$ & $0.12^{*}$ & -6.3 & 3.6 & 0.06 & $-0.06^{*}$ & 0.72 & -0.26 & 14.37 & 20.82 & -0.29 & -0.01 & 4.23 & 5.335 \\
\hline $\begin{array}{l}\text { N10/ } \\
\text { N2 }\end{array}$ & -0.33 & -5.7 & -9 & 0.08 & 0.11 & -0.57 & -0.95 & 30.73 & $9.22^{*}$ & 0.33 & 0.03 & $4.83^{*}$ & $83.34^{*}$ \\
\hline $\mathrm{D} / \mathrm{N} 4$ & -0.34 & -2.4 & -5.6 & 1.33 & 0.18 & -0.27 & -0.76 & 30.5 & 14.96 & -0.09 & 0.03 & 0.63 & -39.7 \\
\hline $\mathrm{K} / \mathrm{N} 4$ & -1.54 & -6.9 & 8.8 & -0.67 & 0.54 & $-2.03^{*}$ & -1.1 & 16.47 & 47.06 & 0.68 & 0.28 & 9.13 & 45.34 \\
\hline N1/N4 & 2.46 & -4.6 & 3.8 & 1.84 & 0.38 & -2.14 & -0.89 & -7.1 & 27.3 & 0.04 & 0.11 & 6.03 & 8.34 \\
\hline $\begin{array}{l}\text { N10/ } \\
\text { N4 }\end{array}$ & -0.27 & -9.4 & -2 & 1.34 & 0.32 & -1.94 & -0.72 & -13.3 & 19.12 & 0.66 & 0.04 & 7.23 & 43,335 \\
\hline N2/N4 & -0.6 & -2.6 & 0.8 & 1.2 & 0.35 & 0.77 & -0.73 & 2.77 & 20.42 & 0.11 & 0.05 & 5.23 & 48.34 \\
\hline $\mathrm{D} / \mathrm{S}$ & $-3.17^{*}$ & -1.9 & $-28.8^{*}$ & 1.62 & 0.81 & 1.99 & -1.89 & 18.67 & 43.56 & -0.418 & -0.26 & -1.47 & $-156^{*}$ \\
\hline $\mathrm{K} / \mathrm{S}$ & -1.82 & $-26.9^{*}$ & -15.8 & -7.54 & 0.57 & -0.96 & -1 & -12.43 & 23.42 & 0.11 & -0.04 & 10.83 & $-224^{*}$ \\
\hline $\mathrm{N} 1 / \mathrm{S}$ & -1.93 & $-22.4^{*}$ & -22.2 & -0.12 & 0.18 & -0.74 & -0.42 & 30.87 & $57.32^{*}$ & -0.4 & 0.07 & 5.03 & $-123^{*}$ \\
\hline N10/S & $-3.19^{*}$ & -30.4 & -22.2 & -0.84 & 0.84 & -2.21 & -1.39 & -0.53 & 44.6 & 0.49 & -0.09 & 2.43 & -1.67 \\
\hline $\mathrm{N} 2 / \mathrm{S}$ & -2.54 & -16.8 & -21 & -1.23 & 0.27 & -2.14 & -1.17 & 17.17 & 38.2 & -0.788 & -0.21 & 1.03 & -53.7 \\
\hline $\mathrm{N} 4 / \mathrm{S}$ & -2.88 & -5.6 & -8.7 & 2.05 & 0.79 & -1.88 & 0.78 & 32.57 & 47.62 & -0.35 & -0.2 & -0.87 & -88.7 \\
\hline
\end{tabular}

Significance tested at $P=0.05$, ${ }^{*}$ significant at $P \leq 0.05$. S. O. V.: source of variation, 1000 gwt: 1000 grain weight, $50 \%$ f: days to $50 \%$ flowering, Grl: grain length, Grw: grain width, Pht: plant height, Pwt: panicle weight, Pnl: panicle length, Ti: total tillers, Fs: fertile spikelets, Ufs: unfertile spikelets, Ut: unproductive tillers, g: grams, cm: centimetres, $\mathrm{t} \cdot \mathrm{ha}^{-1}$ : tonnes per hectare, and $\mathrm{mm}$ : millimetres.

there is high magnitude of SCA effect in addition to high GCA effects from both or at least one of the parents. Similar findings were reported by Dorosti and Monojjen [5], Kumar et al. [6], and Thakare et al. [7]. It is not a must that parents involved in making of crosses are of high GCA effects to get desirable SCA effects. Reason being that there are positive interactions between cytoplasmic genes and nuclear genes becoming more important than interactions between nuclear genes alone.

3.5. GCA and SCA Variance Components Ratios. The current investigation found out that the GCA variance-to-SCA variance ratio was less than one for all traits under study except for biomass, which had more than one GCA
variance-to-SCA variance ratio (Table 5). More than one GCA variance-to-SCA variance ratio indicates additive gene action for biomass, while less than one GCA variance-to-SCA variance ratio indicates that additive gene action prevailed for all other traits. Preponderance of additive gene action over nonadditive gene action is ideal for exploitation in breeding. This study revealed nonadditive gene action for days to 50\% flowering. Similar findings were reported by Upadhyay and Jaiswal [8] and Kumar et al. [9], though Contrary findings were reported by Patil et al. [10] and Salgotra et al. [11] who reported additive gene action for days to $50 \%$ flowering. This study also revealed nonadditive gene action for yield which was similar to Bhadru et al. [12] but differed with reports by Upadhyay and Jaiswal [8], Gnanasekaran [13], and 
TABLE 5: Ratios of GCA variance to SCA variance for grain yield and agronomic traits at Bahari and KALRO Mtwapa during 2018 long rain season.

\begin{tabular}{lcccc}
\hline Characters & $\sigma^{2}$ gca & $\sigma^{2}$ sca & $2 \sigma^{2}$ GCA $/\left(2 \sigma^{2}\right.$ GCA $+\sigma^{2}$ SCA $)$ & Gene action \\
\hline Yield & 0.518 & 1.964 & 0.2637 & Nonadditive \\
Days to 50\% flowering & 67.946 & 74.144 & 0.9164 & Nonadditive \\
Plant height & 76.91 & 110.873 & 0.6936 & Nonadditive \\
Number of tillers & 1.411 & 4.23 & 0.8335 & Nonadditive \\
Unproductive tillers & 0.024 & 0.172 & 0.0132 & Nonadditive \\
Panicle length & 0.785 & 1.818 & 0.431 & Nonadditive \\
Panicle weight & 0.141 & 1.343 & 0.104 & Nonadditive \\
Fertile spikelets & 532.872 & 734.75 & 0.7252 & Nonadditive \\
Unfertile spikelets & 128.089 & 223.07 & 0.574 & Nonadditive \\
Grain length & 0.172 & 0.236 & 0.7288 & Nonadditive \\
Grain width & 0.027 & 0.031 & 0.87 & Nonadditive \\
1000 grain weight & 11.538 & 12.285 & 0.939 & Nonadditive \\
Biomass & 4620.952 & 8.949 & 916.258 & Additive \\
\hline
\end{tabular}

Kumar et al. [9] that showed additive gene action for yield.

This study further revealed nonadditive gene action for plant height and unfertile spikelets. Nonadditive gene action was also reported in plant height by Patil et al. [10] and Dorosti and Monajjem [5]. Nonadditive gene action in unfertile spikelets was reported by Hassan et al. [14] and Nagaraju et al. [15]. The findings in this study agree with their reports. However, contradiction of this finding's additive gene action in plant height was reported by Updhayay and Jaiswal [8], Gnanasekaran et al. [13], and Kumar et al. [9] which reported additive gene action for unfertile spikelets. Biomass inheritance was attributed to additive gene action, though not much has been reported on nature of gene action in biomass. Discrepancies in these reports are attributed to differences in materials used. Thus, findings from one population cannot be extrapolated to another population. Gene action must be estimated for every population.

\section{Conclusion}

In conclusion, from GCA effects, best parents' combiners were Dourado Precoce for days to 50\% flowering, while Supaa and Komboka were best parents for yield. Based on SCA effects, the best performing crosses in terms of yield were $\mathrm{D} / \mathrm{S}$ and $\mathrm{N} 10 / \mathrm{S}$, the in terms of days to $50 \%$ flowering was D/N1. Based on GCA variances, the findings were that additive gene action effects were found in biomass, while nonadditive gene action was obtained in all the other traits under study.

From the findings in this study, we recommend as follows.

Best parents' combiners were Dourado Precoce for days to $50 \%$ flowering, and Supaa and Komboka for yield can be utilized in hybrid and hybridization studies to generate better genotypes. Promising crosses were D/N1 for days to $50 \%$ flowering, and D/S and N10/S for yield can be further developed and extensively tested for stability and superiority before eventual release to farmers.

\section{Data Availability}

Raw data are available from the corresponding author upon request.

\section{Conflicts of Interest}

The authors declare no conflicts of interest regarding the publication of this paper.

\section{References}

[1] MoA, "National rice development strategy (NRDS 2008-2018). P. 35. Modern cultivars," Plant Breeding, vol. 108, pp. 1-11, 2009.

[2] 2018, http://www.datascience.co.ke/food-security-in-kenya/.

[3] B. Griffing, "Concept of general and specific combining ability in relation to diallel crossing systems," Australian Journal of Biological Sciences, vol. 9, no. 4, pp. 463-493, 1956.

[4] IRRI, Standard Evaluation System for Rice, International Rice Research Institute, Los Baños, Philippines, 2013.

[5] H. Dorosti and S. Monajjem, "Gene action and combining ability for grain yield and yield related traits in rice (Oryza sativa L.)," Journal of Agricultural Sciences, vol. 9, no. 3, pp. 100-108, 2014.

[6] P. Kumari, H. K. Jaiswal, and S. A. Waza, "Combining ability and heterosis for yield, its component traits and some grain quality parameters in rice (Oryza sativa L.)," Journal of Applied and Natural Science, vol. 6, no. 2, pp. 495-506, 2014.

[7] I. S. Thakare, A. L. Patel, and A. M. Mehta, "Line $\times$ tester analysis using CMS system in rice (Oryza sativa L.)," The Bioscan, vol. 8, no. 4, pp. 1379-1381, 2013.

[8] M. N. Upadhyay and H. K. Jaiswal, "Combining ability analysis for yield and earliness in hybrid rice (Oryza sativa L.). Asian journal of crop science, 7: 81-86. using a double haploid population," Breeding Science, vol. 52, pp. 309-317, 2015.

[9] S. Kumar, H. B. Singh, and J. K. Sharma, "Combining ability analysis for grain yield and other associated traits in rice," Oryza, vol. 44, pp. 108-114, 2007.

[10] P. R. Patil, V. H. Surve, and H. D. Mehta, "Line $\times$ tester analysis in rice (Oryza sativa L.)," Madras Agriculture Journal, vol. 99, no. 4-6, pp. 210-213, 2012.

[11] R. K. Salgotra, B. B. Gupta, and P. Singh, "Combining ability studies for yield and yield components in Basmati rice," Oryza, vol. 46, pp. 12-18, 2009.

[12] D. Bhadru, R. D. Lokanadha, D. L. Reddy, and M. S. Ramesha, "The effect of environment on combining ability and heterosis in hybrid rice," Greener Journal of Agricultural Sciences, vol. 3, no. 9, pp. 669-686, 2013. 
[13] M. Gnanasekaran, P. Vivekanandan, and C. Muthuramu, "Combining ability and heterosis for yield and grain quality in two-line rice (Oryza sativa L.) hybrids," Indian Journal of Genetics, vol. 66, no. 1, pp. 6-9, 2006.

[14] M. J. Hasan, M. U. Kulsum, M. Hossain, M. H. Manzur, M. R. Mustafizur, and R. N. M. Farhat, "Combining ability analysis for identifying elite parents for heterotic rice hybrids," Academia Journal of Agricultural Research, vol. 3, no. 5, pp. 70-75, 2015.

[15] C. Nagaraju, S. M. Reddy, K. T. Sandeep, K. Hariprasad, and P. Sudhakar, "Gene action and combining ability analysis for grain yield and quality components in rice progressive agriculture," Journal of Plant Breeding and Genetics, vol. 15, no. 1, pp. 35-40, 2015. 